La radio como construcción de subjetividades. Diálogos entre dispositivos escolares y de salud

\title{
La radio como construcción de subjetividades. Diálogos entre dispositivos escolares y de salud
}

\author{
The radio as a construction of subjectivities. Dialogues between \\ school and health devices
}

Mariano Molina marianomolina.radio@gmail.com http://orcid.org/0000-0002-5956-6602

Facultad de Periodismo y Comunicación Social; Universidad Nacional de La Plata (Argentina)

Ayelen Correa Garabello ayecorrea@gmail.com http://orcid.org/0000-0002-1653-2672 Instituto de Investigaciones en Comunicación; Facultad de Periodismo y Comunicación Social; Universidad Nacional de La Plata/ Comisión de Investigaciones Científicas de la Provincia de Buenos Aires (Argentina)

\section{Resumen}

En cada momento histórico y sociocultural se instalan determinadas tecnologías y dispositivos que configuran diversas subjetividades; en consecuencia la vida social y los cuerpos están siendo performados según las coordenadas de cada época y sus instituciones.

Este trabajo pretende observar cómo fueron cambiando algunos elementos de esa trama y sus reconfiguraciones. Específicamente nos proponemos reflexionar sobre la relación de los 
cuerpos y las subjetividades mediadas por experiencias y procesos de comunicación/educación en dispositivos escolares y de salud.

Palabras claves: Comunicación; educación; dispositivos.

Abstract

In each historical and sociocultural moment certain technologies and devices are installed that configure diverse subjectivities, consequently social life and bodies are being performated according to the coordinates of each epoch and its institutions.

This work aims to observe how they were changing some elements of that plot and its reconfigurations. Specifically, we propose to reflect on the relationship of bodies and subjectivities mediated by experiences and processes of communication / education in school and health devices.

Keywords: Communication; education; devices.

En primer lugar, es importante aclarar que entendemos por dispositivo a una trama de diferentes significantes que reúne intervenciones que van desde políticas públicas hasta prácticas concretas y situadas de sujetos, lenguajes y recursos atravesados por el saber, el poder, las subjetividades e identificaciones. Deleuze, vía Foucault, expresa que:

Los dispositivos tienen, pues, como componentes líneas de visibilidad, de enunciación, líneas de fuerzas, líneas de subjetivación, líneas de ruptura, de fisura, de fractura que se entrecruzan y se mezclan mientras unas suscitan otras a través de variaciones o hasta de mutaciones de disposición (Deleuze, 1990).

Asimismo, hablar de comunicación/educación es hacerlo desde una actitud reflexiva que invita a pensar las conexiones entre la formación de sujetos y la producción de sentidos en el entramado de la cultura y la política.

Sería posible sostener que existen dos caras de esa articulación entre formación de sujetos (objeto de la educación) y producción de sentidos (objeto de la comunicación): 
1. Una cara es la que se percibe en el análisis de cómo los equipamientos culturales producen disposiciones subjetivas: históricamente considerado, cada nuevo equipamiento en el marco de una cultura (como lo son, por ejemplo, los medios y las nuevas tecnologías, pero también las distintas instituciones u organizaciones educativas) opera modificaciones en las disposiciones prácticas, las competencias, las percepciones, de los sujetos.

2. Otra cara es la que nos permite visualizar la articulación entre interpelaciones y reconocimientos (o no reconocimientos): existen "invitaciones" a ser de determinadas maneras, que funcionan como interpelaciones o "modelos de identificación" (sostenidos por los medios, por las escuelas, por grupos generacionales, políticos, sexuales, etc.), frente a los cuales los sujetos nos reconocemos (las asumimos, las hacemos propias, queremos ser lo que se nos invita a ser, las incorporamos, incluso las llevamos a la práctica) o no (Huergo, 1997: 12).

\section{La escuela como institución pública y tecnología}

Es interesante la reflexión que realiza Paula Sibilia acerca de las necesidades de la existencia de una institución como la escuela en el presente, y fundamentalmente nos ayuda a pensar la escuela como una tecnología perteneciente a una época que configuraba determinadas subjetividades y cumplía con fines sociales muy puntuales, al menos en apariencia.

Al observarla bajo el prisma historiográfico, esa institución gana los contornos de una tecnología: se la puede pensar como un dispositivo, una herramienta o un intrincado artefacto destinado a producir algo. $Y$ no cuesta demasiado verificar que ese aparataje se está volviendo gradualmente incompatible con los cuerpos y subjetividades de los chicos de hoy. La escuela sería, entonces, una máquina anticuada (2012: 11).

El planteo de Sibilia nos ayuda a abordar el tema, aunque para poder complejizarlo un poco más debemos agregar que la escuela no sólo es una herramienta y un dispositivo tecnológico, en este caso con muchos rasgos anticuados. La escuela también es una institución social y su importancia va más allá de ser una herramienta tecnológica. En nuestro país pertenece a un principio que nace, incluso, desde la primera Constitución Nacional en 1853. En al artículo 5 de esa Constitución ya se dejaba en claro que la educación era una obligación de los Estados provinciales y nacional. Treinta años más tarde, en 1884, la Ley 1420 ratificaba estas obligaciones estatales. Es decir que podríamos establecer una tensión que recorrerá probablemente la vida del sistema educativo argentino: por un lado la obligación estatal y el 
derecho ciudadano, y por otro, una de las herramientas de implementación, en este caso la escuela, que es además una herramienta casi universal de las sociedades occidentales. La pedagoga Adriana Puiggrós la denomina "esa vieja vaca sagrada", a la cual defiende como herramienta necesaria de inclusión y construcción de ciudadanía.

Pero como dijimos anteriormente, no es un propósito realizar una genealogía exhaustiva. Simplemente intentamos ver algunos rasgos de un dispositivo y poner en diálogo algo de la historia de esta institución y tensionarla, complejizarla, para poder llegar al tema central que queremos compartir en este escrito: reflexionar acerca de lo que sucede en algunas experiencias de comunicación/educación dentro del sistema escolar, transitando por el dispositivo denominado escuela, así como en otros espacios de formación no escolar, como el dispositivo de salud (mental). Ambos conectados por compartir experiencias comunicacionales vía el recurso de la radio.

\section{Tecnologías y neutralidades}

Las herramientas que produce la tecnología no son neutrales, aunque parte del sentido común construido hegemónicamente exprese lo contrario. Fundamentalmente porque ese sentido común se construye y promueve desde las cosmovisiones que detentan quienes son impulsores del orden económico y social capitalista. Al respecto, Sibilia nos dice algo muy concreto sobre los dispositivos tecnológicos, ejemplificando con algunos de uso masivo en los últimos años:

(...) ni las computadoras, ni Internet ni los teléfonos móviles son recursos "neutrales" como suele decirse, cuya eficacia dependería de la utilización que se le dé. Al contrario, como ocurre con todas las máquinas, estas no son ni buenas ni malas, pero tampoco se puede asumir que son neutras. Cargan consigo toda una serie de valores y modos de uso que están implícitos, por más que siempre exista cierto grado de flexibilidad, agenciamiento, experimentación y apropiación por parte de sus usuarios, pero eso no significa que no posean su propia materialidad y su impronta bastante característica (2012: 184).

Atenderemos a algunas herramientas utilizadas en las instituciones escolares durante gran parte del siglo XX y en la actualidad, como pueden ser la tiza y el pizarrón. ¿Qué nos dicen la tiza y el pizarrón? ¿De qué tipo de organización nos hablan y qué subjetividades subyacen en 
su utilización? O al decir de Sibilia: ¿qué valores cargan y qué modos de uso están implícitos en su aplicación?

La tiza es parte de una tecnología que nos ayuda a escribir, pero también es complemento esencial del pizarrón, que marca una disposición del aula y los cuerpos, establece un frente y un adelante donde escribe el/la docente y las y los estudiantes copian (reproducen), organiza las miradas y conforma la disposición áulica. Estas escenas, que persisten en la actualidad, se configuraron en un momento que podríamos denominar cultura letrada, donde en las clases se ponía en juego una forma del saber, la lectura ocupaba gran espacio temporal en la vida cotidiana, las denominadas clases magistrales de las y los docentes era un formato característico, lo que implicaba también dejar en claro de dónde provenía el conocimiento.

Estos dispositivos también promueven una configuración de las subjetividades en determinado momento histórico. La escuela, que es una de las instituciones claves de la sociedad disciplinaria, contiene estos dispositivos y se propone moldear algunas acciones de las y los sujetos. El dispositivo escolar ha sido descrito largamente, pero en este caso nos motiva la posibilidad de reflexionar sobre algunas transformaciones que se expresan en la sociedad contemporánea, junto a prácticas relacionadas al campo de la comunicación, que promueven otras instancias de reconocimiento, interpelación y participación.

En la sociedad disciplinaria algunos de los dispositivos relacionados al mundo de la escuela eran el pizarrón, la tiza, el libro o la carpeta y la construcción de subjetividades imponía una disciplina que permitía estar dos o más horas seguidas atendiendo una clase o leyendo en silencio un libro. En las sociedades contemporáneas algunos de esos dispositivos cambiaron hacia las computadoras, tablets o teléfonos inteligentes y las subjetividades que se construyen por fuera de la escuela tienen una presencia igual o mayor que la del establecimiento educativo.

Para ejemplificar, podemos observar la poca disposición de las y los estudiantes a clases largas, pérdida de concentración, la ansiedad por conectarse a alguna red social o la poca voluntad de lectura con textos medianamente extensos. Estos fenómenos tienen dos características que quisiéramos destacar. Por un lado, son fenómenos que construyen las subjetividades de la sociedad contemporánea o gran parte de ella, pero además se imponen mayoritariamente desde afuera de los establecimientos educativos, lo que lleva a la pérdida del lugar central que ocupaban en la sociedad disciplinaria.

Frente a estos fenómenos que atraviesan a la sociedad, la escuela y sus prácticas también entran en debate y se tensionan, porque más allá de los lamentos por la falta de atención (que atraviesa a niños, niñas, adolescentes y adultos), lo cierto es que hay búsquedas de nuevas 
iniciativas que puedan acompañar y poner en debate estas nuevas subjetividades. Quizás uno de los fenómenos de la época sea que las prácticas educativas hoy tienen más posibilidades de generar prácticas disruptivas que en el pasado, precisamente porque la función disciplinadora y constitutiva de la subjetividad viene desde las paredes externas de esa vieja institución de encierro.

Y es paradójico que vamos a analizar lo que sucede en las prácticas de radio, que es precisamente- un medio de comunicación viejo y en algunos casos en desuso. Pero las prácticas radiales en dispositivos de educación y salud nos llevan a reflexionar acerca de procesos que pueden tener horizontes de una construcción subjetiva diferente a la hegemónica, donde la vieja tecnología se reconfigura y tiene una vigencia interesante de observar.

\section{El acto de la palabra}

Los sujetos estamos siendo mediados por el lenguaje. Lacan dice que es en el encuentro de las palabras con el cuerpo donde algo se dibuja. Lenguaje que construye una realidad efectiva en la medida que existe un deseo y una creencia en lo que se percibe; podemos decir entonces que somos, estamos y hacemos según los recortes de la realidad que vamos eligiendo. Así, el proceso de subjetivación del mundo es un trabajo sobre la realidad. Lacan nos invita a abordarlo desde el síntoma como nudo del sujeto, donde toda la experiencia subjetiva es contenida y soportada por diversos elementos que se cruzan y entrelazan. Desde esta línea, Byung-Chul Han expresa que la voz se hace presente como ánimo que devela en la estructura del yo lo diferente, lo extraño a sí mismo.

La voz es algo que regresa del otro mundo, una reaparecida. Lo que fue excluido y reprimido regresa como voz. La negatividad de la negación y la represión es constitutiva de la voz. En la voz regresa el contenido psíquico reprimido (Byung-Chul Han, 2016: 83).

Quizás desde acá se puede pensar a la experiencia radial como caja de resonancia donde los sujetos hacen algo con la voz. Ahora bien ¿qué dicen cada uno en sus relatos? ¿de qué dan cuenta? Como sentidos discursivos dan cuenta de la sublimación de la satisfacción, de esas palabras que se sueltan y que al ponerse en común, esas historias, relatos, saberes e ideas 
posibilitan el encuentro con otras y otros, y en esta relación lo singular se templa y afina, y al hacerlo se constituye.

Encuentro que se da también desde el diálogo con otras y otros donde está presente el arte de escuchar, como quien contempla y contiene algo del decir del otro y en ese acto de "amabilidad" y "paciencia" (Byung-Chul Han) el sujeto se vacía de contenido propio para ocuparse del otro como oyente.

Este proceso de comunicación/educación es pensado como práctica política, donde los/as sujetos se interpelan en la voluntad de encontrarse, escucharse, hablarse, conocerse; y en esa predisposición redescubren el tiempo del otro/a, en tanto otro/a, así como algo de lo singular. En este sentido Byung-Chul Han dice que la escucha:

Es una acción, una participación activa en la existencia de otros, y también en sus sufrimientos. Es lo único que enlaza e intermedia entre hombres para que ellos configuren una comunidad (Byung-Chul Han, 2016: 120).

\section{Sonoridades radiales}

El proceso de trabajo en el dispositivo de salud (mental) es una experiencia que va de lo singular a lo colectivo y viceversa. Hay una exploración en la que los sujetos buscan sus propias maneras de estar y hacer, como el lugar y el rol que quieren ocupar. En esa praxis inventan maneras de decir algo, mediadas por la trama socio-cultural y los estatutos del lenguaje radial, a partir de los deseos y saberes que circulan y se ponen en común con otras y otros. Además hay un abordaje sobre lo corporal, lo sonoro y la respiración que focaliza en la voz propia y en las maneras de sacarla hacia afuera, de sentirla, modelarla, objetivarla y soltarla.

La construcción del programa radial pasa por diferentes estaciones. Hay un tiempo de producción donde se ponen en común las ideas y se reflexiona sobre el qué y por qué se quiere decir, y una vez que se acuerdan los temas se investiga sobre las y los invitadas/os al piso de la radio o entrevistadas/os, así como sobre los temas musicales, textos, etcétera. Una vez que está toda la información se cocina el programa; allí entran en juego los criterios estéticos sobre las palabras, los textos, los sonidos, las pausas, los silencios. Cuestiones que también se tienen en cuenta para armar la artística (cortinas, spots y separadores), donde las sonoridades se editan y son acompañadas con efectos, texturas. Hacer radio se convierte 
entonces en un acto artesanal, intersubjetivo donde el estilo y la identidad del programa van mutando en la relación dialógica entre formas, contenidos y sentidos.

Luego está el tiempo de emisión, donde las y los sujetos se sumergen en otros mundos posibles, otros espacios y tiempos comunicacionales, diferentes al sistema de salud tradicional, y en especial la lógica manicomial. La experiencia posibilita que alojen algún deseo, un sentimiento, un pensamiento, una acción, dejando de ser sujetos pasivos de un discurso (como pacientes) para pasar a ser las y los que enuncian. De esta manera el dispositivo funciona como una posibilidad de escuchar otras voces, que quizás rompen con ciertas lógicas naturalizadas por algunos oyentes, y que invitan a conocer otras historias de vidas, otros puntos de vista, otras invenciones, otras estéticas. En este sentido, la figura del educador/a, su planificación y los tiempos del programa son sensibles y flexibles a las condiciones intersubjetivas y contextuales del momento, por lo que la estructura radial está sujeta a las improvisaciones que haya que hacerse en cada salida al aire.

\section{Procesos de aprendizaje en el proceso de radio en el ámbito escolar}

En el proceso de aprendizaje que se desarrolla alrededor de la propuesta radial encontramos elementos distintivos que intentaremos describir y -también- ir conceptualizando o dialogando con algunos autores.

En primer lugar, hay un cambio paradigmático en relación a lo que sucede con el aprendizaje y el conocimiento. Porque si bien las y los docentes son los iniciadores de un proceso de trabajo y quienes dan el impulso inicial, luego la propuesta radial está abierta al trabajo colectivo y, entonces, el proceso pedagógico comenzará a transitar por algunos caminos no previstos.

Las y los docentes saben que quieren realizar una experiencia radial, asumiendo el desafío del proceso pedagógico que incluye la salida al aire de uno o más programas, pero hay muchos interrogantes que se van a ir dilucidando durante el proceso. No todo está planificado en detalle y esa incógnita sobre el proceso o ausencia de seguridad sobre el desarrollo completo, pone en juego otras alertas y nuevos desafíos.

Las y los estudiantes deben asumir el compromiso de la propuesta y cuando esto sucede, comienza el proceso de trabajo más intenso y significativo de conocimiento y aprendizajes. El adulto-docente cumple la función iniciadora de guía que inicia el proceso de aprendizaje, pero luego es un intercambio de conocimientos y aprendizaje mutuos para ir desarrollando una propuesta colectiva propia y particular. Probablemente, sin haber conceptualizado previamente, 
se produce lo que Paulo Freire definió sobre el aprendizaje colectivo entre estudiantes y docentes y las diferencias entre la educación bancaria y la educación problematizadora.

\begin{abstract}
De este modo, el educador ya no es solo el que educa sino aquel que, en tanto educa, es educado a través del diálogo con el educando, quien, al ser educado, también educa. Así, ambos se transforman en sujetos del proceso en que crecen juntos (...) mientras la práctica "bancaria", como recalcamos, implica una especie de anestésico, inhibiendo el poder creador de los educandos, la educación problematizadora, de carácter auténticamente reflexivo, implica un acto permanente de descubrimiento de la realidad (Freire, 1968: 85-
\end{abstract} 86).

En los procesos de trabajo, la disposición de los cuerpos en el aula empieza a modificarse respecto de lo que se conoce como el aula tradicional. Las alumnas y alumnos trabajan en grupos, se re-distribuyen por el aula, algunos pueden salir a buscar información a la biblioteca de la escuela o realizar entrevistas, otros escuchan algunas grabaciones, editan, planifican, discuten, debaten, escriben. La escena se parece mucho a algo que desde afuera puede ser difícil de comprender. Si un supervisor de mediados de siglo XX (y no tan lejano también) pasa por afuera del aula y desde una ventana o la puerta entreabierta observa los comportamientos, probablemente no entienda lo que está sucediendo y se preocupe por la falta de orden.

Esta escena la podríamos denominar "elogio del ruido", porque es una práctica pedagógica necesaria para un proceso de aprendizaje colectivo de estas características. Sin ruido, no hay creación colectiva y sin creación colectiva no hay aprendizaje y por lo tanto, ausencia de educación problematizadora en esta propuesta pedagógica.

Este proceso que describimos lleva un tiempo de trabajo que dura semanas o meses, donde incluso la tiza y el pizarrón son dispositivos secundarios. En esas instancias de trabajo, docentes y estudiantes van pasando por varias etapas muy significativas. Podemos asegurar que el proceso pedagógico de trabajo con el dispositivo radial configura una relación temporal que limita las ansiedades y las relaciones inmediatas, porque imprescindiblemente lleva tiempos de reflexión, planificación y acción contrarios a las dinámicas hegemónicas de la cotidianeidad.

\title{
La escena no ordinaria
}


Hay una interesante relación que se produce en el momento mismo de la salida al aire. Hacer un programa de radio en vivo impone una situación extraordinaria en relación a la vida cotidiana y las instancias de aprendizaje áulico. En ese momento los cuerpos se predisponen alrededor de una mesa, todos se pueden mirar a la cara (y se miran), es necesario que cada sujeto escuche atentamente lo que sucede, preste atención a las palabras del otro, no hay superposición de voces, cada cual espera su tiempo para hablar, pero también respeta los tiempos ajenos. Pensemos en qué escenas de la vida cotidiana nosotros mismos estamos en situaciones similares y será difícil describir muchas.

La voz es necesariamente una relación con el otro, con lo externo e implica formas de relacionamiento. Hacer un programa de radio es, entonces, una experiencia de orden educativo y corporal.

La comunicación digital es muy pobre de mirada y de voz. Los enlaces y las interconexiones se entablan sin mirada ni voz. En eso se diferencian de las relaciones y los encuentros, que requieren la voz y la mirada. Es más, son experiencias especiales de la voz y la mirada. Son experiencias corporales (Byung-Chul Han, 2016: 92).

Además, la posibilidad de expresión es fundamental en el reconocimiento del sujeto como actor del proceso. A mediados del siglo XX Celestin Freinet afirmaba que "Los niños deben escribir para ser leídos, no escribir por escribir o porque se los ordena el maestro". En esta misma línea conceptual podemos afirmar que tienen que producir contenidos radiofónicos para que sean escuchados. La experiencia de atravesar un programa de radio es una construcción de su identidad y un espacio de reconocimiento a sus producciones.

Cuando un estudiante puede hablar y reflexionar sobre lo que estuvo trabajando a lo largo de semanas o meses, entonces podemos afirmar que hubo un proceso de aprendizaje. La posibilidad de que el sujeto pueda expresar con sus propias palabras y conocimientos (a veces extensos y a veces simples) sobre la temática que está abordando, es una muestra del proceso pedagógico-comunicacional realizado, aunque en algunas ocasiones no cumpla con formas estandarizadas de la evaluación.

En el programa de radio se produce otro fenómeno importante, que es el dejar de lado los dispositivos smartphone, a veces por sugerencias y a veces por decantación. La única utilización posible del dispositivo teléfono inteligente es la imagen, producir fotos que retraten ese instante experiencial. Pero en la hora de aire en el estudio las necesidades de conectividad 
quedan en un plano inferior, porque es la experiencia concreta que se vive la que ocupa toda la atención corporal y mental.

Todos estos elementos que estamos nombrando como la escucha, la disposición de los cuerpos para observarse mutuamente, la paciencia de esperar cada uno su espacio, el acceso a la palabra o la producción de conocimiento son elementos distintivos de un proceso de comunicación/educación que produce experiencia.

\section{Aproximaciones a la experiencia}

El proceso de trabajo desde una institución escolar o en el campo de la salud con el dispositivo radial deviene en experiencia. ¿Qué tipo de experiencia se expresa en este proceso de comunicación/educación?

Intentaremos ir abordando algunas definiciones que queremos compartir y que de algún modo, van sistematizando las prácticas vivenciadas.

La experiencia social, según la define Berta Orozco Fuentes, es una característica importante ya que los saberes y aprendizajes socialmente significativos son aquellos que se generan a través de la experiencia social, que modifican a el/la sujeto, enriqueciendo su capital cultural y diferenciándose de los conocimientos repetidos.

Como dijimos anteriormente, sabemos dónde se inicia el proceso pero no se sabe cómo terminará. Esa característica es excepcional para la educación. Los estudiantes viven una experiencia significativa. Francis Bacon afirmaba que "El conocimiento de la experiencia es intransferible, pero es comunicable". El interrogante sobre las formas de transmitir la experiencia también se hace presente.

Pero para que la experiencia produzca sucesión, importa que sea compartible... pues la experiencia se transmite en forma de conocimientos. No importa si la forma que ellos adquieren es el discurso científico, mítico u otros lenguajes, sino que éstos transporten información construida en otra circunstancia (Puiggrós, 2004: 71).

La experiencia es una vivencia necesaria para luego poder conceptualizar o racionalizar. En este sentido Michelle Maffesoli afirma que "Antes de cualquier racionalización existe la vivencia. Uno de los rasgos de la vivencia es la Experiencia". 
Desde un punto de vista, la experiencia subjetiva, en ciertas condiciones, puede constituir una serie de saberes, aunque nadie repare en ellos, aunque no los reclame ninguna nueva generación e incluso aunque el sujeto no sepa transmitirlos. Me refiero a los sujetos, no solamente a los individuos, porque un grupo social puede compartir experiencias que no son transmisibles o que no encuentran receptores. No viene al caso, tampoco, la calidad o la utilidad de esa información para quienes eventualmente pueden ser sus receptores. No es necesario que se trate de una situación compartible, o bien puede ser imposible compartirla a causa de obstáculos que provienen de la sociedad, de la cultura o del momento histórico. La experiencia no es, a fuerzas, socialmente productiva (Puiggrós, 2004: 71)

Desde este concepto, es importante destacar las experiencias producidas más allá de las posibilidades de amplificación, que son también producto de luchas pedagógicas y políticas por las que atravesamos permanentemente.

\section{Experiencia performativa}

Lenguaje radiofónico pensado como acto estético que va desde la experiencia del sujeto al objeto como cosa, como aquello que se suelta en un hacer creativo. Creación no como un objeto nuevo con elementos nuevos, más bien desde la idea de un objeto creado por nuevos interlocutores que en la medida en que los sujetos hacen algo con el objeto significante se inventan a sí mismos. Invención que se desprende de la intersección entre sujeto-objeto. Por ejemplo, uno de los participantes del dispositivo de salud expresa que el recurso de la intertextualidad "me llevó a encarar otro tipo de estructura dialéctica", hacia otras maneras de reflexionar y hacer por otros campos, temas y formatos, a "aprender a escuchar y aprender a formular ideas, [a] expresar la procesión que uno lleva por dentro"; en un estar siendo mediado por la relación con algo, desde el entre-otros, que lo moviliza. Ese <me $>$ tiene que ver con lo que le pasa a él, desde él, como lugar de la experiencia (Skliar y Larrosa, 2009: 15), en sus ideas, en sus sentimientos, en sus palabras, en su saber, en su intención de hacer algo. Experiencia entendida según Larrosa desde tres principios: reflexibilidad, subjetividad y transformación.

Como principio de reflexividad, en la relación entre la comprensión y la expresión de dicha comprensión, desde otros puntos de vista que con-muevan a pensar(nos) desde otras coordenadas posibles. Juego que se da en un movimiento de ida y vuelta: 


\begin{abstract}
Un movimiento de ida porque la experiencia supone un movimiento de exteriorización, de salida de mí mismo, de salida hacia fuera, un movimiento que va al encuentro con eso que pasa, al encuentro con el acontecimiento. $Y$ un movimiento de vuelta porque la experiencia supone que el acontecimiento me afecta a mí, que tiene efectos en mí, en lo que yo soy, en lo que yo pienso, en lo que yo siento, en lo que yo sé, en lo que yo quiero, etc. (Skliar y Larrosa, 2009: 16).
\end{abstract}

Desde el principio de subjetividad como experiencia subjetiva y singular, donde el sujeto está pre-dispuesto a que algo le afecte, "Se trata de un sujeto abierto, sensible, vulnerable, ex/puesto" (Skliar y Larrosa, 2009: 16).

Y el principio de transformación como aquel que:

hace la experiencia de su propia transformación. De ahí que la experiencia me forma y me transforma. De ahí la relación constitutiva entre la idea de experiencia y la idea de formación. De ahí que el resultado de la experiencia sea la formación o la transformación del sujeto de la experiencia. De ahí que el sujeto de la experiencia no sea el sujeto del saber, o el sujeto del poder, o el sujeto del querer, sino el sujeto de la formación y la transformación. De ahí que el sujeto de la formación no sea el sujeto del aprendizaje (al menos si entendemos aprendizaje en un sentido cognitivo), ni el sujeto de la educación (al menos si entendemos educación como algo que tiene que ver con el saber), sino el sujeto de la experiencia (Skliar y Larrosa, 2009: 16).

En ese saber hacer y hacer, ese saber decir y decir, mediado por el lenguaje radial, algo cambió, y en su acción la palabra hablada performa al sujeto en el acto de narrarse, como caja de resonancia donde la carne deviene cuerpo, como expresión "que manifiesta y define al mismo tiempo" la idea de un yo, de un sujeto capaz de "articularse a sí mismo" (Taylor, 1931: 396). Y es ahí cuando el acto estético pasa a tener un lugar central en el sujeto, como fuerza que manifiesta un real existir con otras y otros, no como reproducción, sino a través de la interlocución con otras y otros y de exponer el cuerpo frente a otras y otros, como "constitución de la cultura oral" (Huergo y Fernández, 2000: 121), propia de lo popular diría Kusch.

Así el proceso de subjetivación muestra el grado de compromiso geográfico de nuestro ámbito vital (Kusch, 1956), de un sujeto situado y afectado por su geocultura, que ya no se piensa encerrado en sí mismo, quieto, solo, sino que se reconoce en movimiento con otras y otros. Sensibilidad que es fagocitada por la trama de horizontes culturales en tensión y contradicción, 
donde se pone en juego la "imaginación creativa" (Castoriadis), como fuerza que expresa una realidad; no como reproducción, sino como nuevas formas simbólicas de hacerla visible y más cercana; el "imaginario efectivo" (Castoriadis) como la regulación subjetiva de los límites, lo permitido, lo adecuado, lo consensuado; y el "imaginario radical" (Castoriadis) como la posibilidad de inventar nuevas maneras de ser y estar con otras y otros.

Imaginarios que se atan y desatan en el entramado dialógico de quien expresa algo y quien se siente interpelado por ello, desde la intersección entre lo que puede ser "una presencia fugaz porque no está dominada por el sentido de la vista" y como "palabra provocativa de una presencia imaginaria, cargada de sonidos y tejiendo la memoria. Es una palabra que genera sueños" (Huergo y Fernández, 2000: 123). Allí se arma una escena, desde lo que se dice como por lo que no se ve ni se toca pero está presente en las pausas, en los silencios, en los sonidos gravitantes mediados por el poder de la escucha, como un "lenguaje invisible, seductor y sugerente, hasta hacerse ausente" (Idem, 2000: 123). En este sentido:

la Radio provoca un uso de la memoria más allá de su relación cartesiana con el método científico y más cercana a la memoria que caracteriza la oralidad. Una memoria de la palabra escuchada que está más cerca de lo oscuro y confuso que de lo claro y distinto, lo cual hace que se desplace de la racionalidad manipuladora (Ibídem, 2000: 121).

Apalabrarnos posibilita que en el encuentro con otros y otras se pueda, aunque sea por un instante, salirse de uno mismo, poder estar, escuchar e interesarse por otros y otras por "interiorizarme o preocuparme por el pensar o ver lo que le pasa a los demás, [por] involucrame en temas más sociales" (1). Es ahí donde lo político se anuda, donde el vínculo con otros y otras "parece tener cierto poder para crear un espacio comunitario: un lugar de refundación del sentido de una comunidad histórica, capaz de reconstruir la trama de lo popular (...)." (Huergo y Fernández, 2000: 129-130). Recuperar la memoria colectiva desde una oralidad dialógica que irrumpa y supere el individualismo atomizante, que sea sensible a las experiencias situadas de los acontecimientos singulares, como alteridades que constituyen lo plural.

\section{Conclusiones}

Las experiencias de educación/comunicación que hemos analizado nos hablan sobre la posibilidad de descentralizar e inventar, cada vez, los dispositivos. Ya no desde el modelo 
disciplinar trascendente, de normalización y reproductividad, sino más bien desde lo inmanente, en constante movimiento y transformación. Se trata de pensarlos desde el lugar que le damos, qué sentidos le atribuimos y qué prácticas conviven. De reconfigurar los espacios y sujetos desde los modos de existencia, donde "podemos conocer las cosas no sólo por su razón actual sino por todas las relaciones de las que son capaces" (Duschatsky y Aguirre, 2013: 38) en el devenir de lo cotidiano. Es decir, habitar los espacios y contener otras subjetividades posibles creando sentidos que alojen su estar siendo y haciendo. Para esto es necesario construir una relación pedagógica que reconozca los deseos y saberes, y que cuide los lazos vivificantes que posibilitan encuentros, expresiones y aprendizajes.

La territorialización es más tiempo compartido que espacio establecido bajo propiedades que exceden a sus ocupantes; más proceso de construcción de encuentros que reproducción de relaciones preexistentes. Los territorios de los que hablamos son trazados problemáticos, decididos no sobre la base de fantasías imaginarias sino a partir de las fuerzas vivas que piden formas. Los espacios son entidades cerradas que no pueden habitarse de cualquier modo sino de acuerdo con sus propiedades ya definidas. Los territorios, o tal como lo pensamos, los procesos de territorialización designan configuraciones vivas y plausibles de diferenciación (Duschatsky y Aguirre, 2013: 43).

¿Qué puede hacer el recurso radial desde el campo de Comunicación/Educación? En estos dispositivos la experiencia marca y construye horizontes culturales diferentes a los instituidos; así la radio habilita un desplazamiento de los sujetos que, mediados por el lenguaje e impulsados por el deseo de conocer, relacionarse e interpelar a otros y otras, inventan otras formas de sensibilidad y socialidad cercanas a las culturas orales, posibilitando otras maneras de percibir, interpretar, imaginarse y hacer otros mundos. El sujeto, a través de la oralidad dialógica mediatizada por la radio, se performa desde el vínculo con otros y otras, desde el uso de la palabra, el acceso y apropiación de las nuevas tecnologías. Estos microespacios y poderes gestan desde un sentido político-emancipador nuevos sujetos y memorias colectivas. A lo largo de este escrito intentamos reflexionar que, en situaciones concretas, un viejo dispositivo como es la radio puede reconfigurarse en el marco del campo comunicación/educación. Esta tecnología, diseñada para transmitir información direccional, desde una base a infinitas recepciones, ha sido reconceptualizada en acciones particulares que permiten atravesar experiencias significativas en los sujetos, mediante otra interacción, disposición de los cuerpos, formas de acceso a la palabra, la expresión e incluso, construcción de ciudadanía. 
La posibilidad de que estas experiencias significativas para sujetos e instituciones hayan modificado las relaciones entre esos dispositivos y sus modos de intervención en espacios públicos e instituciones -también configuradas en épocas de la sociedad de control- no desconoce lo que afirmamos al inicio. Los instrumentos diseñados por la tecnología no son neutros y por lo tanto están cargados de intenciones, búsquedas, sentidos y construcción de subjetividades. Este principio ordenador nos obliga a reflexionar y a preguntarnos por la historia de los diversos dispositivos y nuestras intervenciones. Creemos que la pregunta permanente es la posibilidad de poder seguir transitando experiencias importantes desde el campo comunicación/educación, asumiendo las tensiones y riesgos que conlleva toda relación entre los sujetos y dispositivos.

\section{Notas}

(1) Palabras de uno de los participantes del dispositivo de salud.

\section{Bibliografía}

Byung-Chul, H. (2016). La expulsión de lo distinto. España: Herder Editorial.

Deleuze, G. (1990). ¿Qué es un dispositivo? En Foucault, filósofo. Argentina: Gedisa Editores.

Duschatsky, S. y Aguirre, E. (2013). Des-armando escuelas. Ciudad Autónoma de Buenos Aires: Editorial Paidós.

Freire, P. (1968). Pedagogía del oprimido. Siglo Veintiuno Editores.

Huergo, J. y Fernández, M. B. (1999). Cultura escolar, Cultura mediática/Intersecciones. Colombia: Universidad Pedagógica Nacional.

Huergo, J. (1997). Comunicación/Educación: ámbitos, prácticas y perspectivas. La Plata: Ediciones de Periodismo y Comunicación.

Kusch, R. (1956). Anotaciones para una estética de lo americano. Identidad, 1, 6-20.

Lacan, J. J. (1984). El Seminario de Jacques Lacan, 3: Las Psicosis (1955-1956). Barcelona: Editorial Paidós Ibérica.

Orozco Fuentes, B. (2006). Aprendizajes socialmente significativos: en diálogo y tensión con los discursos del aprendizaje y las competencias en educación. Ponencia presentada en el Foro 50 años del Colegio de Pedagogía. Facultad de Filosofía y Letras, Universidad Nacional Autónoma de México. 
Puiggrós, A. (2011). De Simón Rodríguez a Paulo Freire. Educación para la integración iberoamericana. Buenos Aires: Ediciones Colihue.

Skliar, C. y Larrosa, J. (2009). Experiencia y alteridad en educación. Rosario: Homo Sapiens Ediciones.

Sibilia, P. (2012). ¿Redes o paredes? La escuela en tiempos de dispersión. Ciudad Autónoma de Buenos Aires: Tinta Fresca Ediciones. 